\title{
Bifurcation Analysis in a Delayed Diffusive Leslie-Gower Model
}

\author{
Shuling Yan, Xinze Lian, Weiming Wang, and Youbin Wang \\ College of Mathematics and Information Science, Wenzhou University, Wenzhou 325035, China
}

Correspondence should be addressed to Weiming Wang; weimingwang2003@163.com

Received 29 September 2012; Accepted 18 November 2012

Academic Editor: Yanbin Sang

Copyright (C) 2013 Shuling Yan et al. This is an open access article distributed under the Creative Commons Attribution License, which permits unrestricted use, distribution, and reproduction in any medium, provided the original work is properly cited.

We investigate a modified delayed Leslie-Gower model under homogeneous Neumann boundary conditions. We give the stability analysis of the equilibria of the model and show the existence of Hopf bifurcation at the positive equilibrium under some conditions. Furthermore, we investigate the stability and direction of bifurcating periodic orbits by using normal form theorem and the center manifold theorem.

\section{Introduction}

The dynamic relationship between predators and their preys has long been and will continue to be one of dominant themes in both ecology and mathematical ecology due to its universal existence and importance. A major trend in theoretical work on prey-predator dynamics has been to derive more realistic models, trying to keep to maximum the unavoidable increase in complexity of their mathematics [1]. In this optic, recent years, the important Leslie-Gower predator-prey model $[2,3]$ has been extensively studied in [4-7]. A modified version of Leslie-Gower predator-prey model with Holling-type II functional response takes the form

$$
\begin{aligned}
& \frac{d H}{d t}=H\left(a_{1}-b H\right)-\frac{c_{1} H P}{k_{1}+H}, \\
& \frac{d P}{d t}=P\left(a_{2}-\frac{c_{2} P}{k_{2}+H}\right),
\end{aligned}
$$

where $H$ and $P$ represent prey and predator population densities at time $t$, respectively. $a_{1}, a_{2}, b, c_{1}, c_{2}, k_{1}$, and $k_{2}$ are positive constants. $a_{1}$ is the growth rate of prey $H . a_{2}$ describes the growth rate of predator $P . b$ measures the strength of competition among individuals of species $H . c_{1}$ is the maximum value of the per capita reduction of $H$ due to $P$, and $c_{2}$ is the maximum value of the per capita reduction of $P$ due to $H$, which is not available in abundance. $k_{1}$ measures the extent to which environment provides protection to prey
$H . k_{2}$ measures the extent to which environment provides protection to the predator $P$.

On the other hand, time delay plays an important role in many biological dynamical systems, being particularly relevant in ecology [1]. For some predator-prey systems, the rate of the prey population depends on the predation of predator in the earlier times [8-14]. The results indicated that delay differential equations exhibit much more complicated dynamics than ordinary differential equations since a time delay could cause a stable equilibrium to become unstable and induce bifurcations.

In this paper, we will focus on the complex dynamics of the delay effect in the extended reaction-diffusion model. The reproduction of the individuals is modeled by diffusion with diffusion coefficients $D_{1}>0$ and $D_{2}>0$ for the prey and predator, respectively. This basic model is described by a system of two partial differential equations:

$$
\begin{gathered}
\frac{\partial H}{\partial t}=H\left(a_{1}-b H\right)-\frac{c_{1} H P(t-\tau)}{k_{1}+H} \\
+D_{1} \Delta H, \quad x \in \Omega, t>0, \\
\frac{\partial P}{\partial t}=P\left(a_{2}-\frac{c_{2} P}{k_{2}+H}\right)+D_{2} \Delta P, \quad x \in \Omega, t>0, \\
\frac{\partial H}{\partial \mathbf{n}}=\frac{\partial P}{\partial \mathbf{n}}=0, \quad x \in \partial \Omega, t>0,
\end{gathered}
$$

where $H=H(t, x), P=P(t, x) . \Delta=\partial^{2} / \partial x^{2}, \Omega$ is a bounded open domain in $\mathbb{R}$ with boundary $\partial \Omega$, $\mathbf{n}$ is the outward unit 


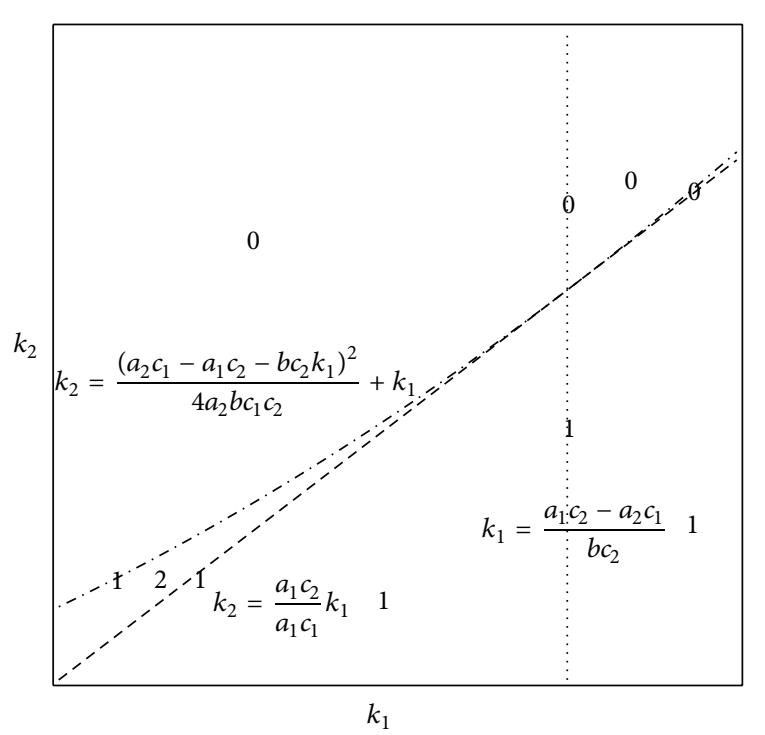

FIGURE 1: The bifurcation diagram displays the distribute of the positive roots; the number indicates the number of positive equilibria.

normal vector on $\partial \Omega$, and homogeneous Neumann boundary conditions reflect the situation where the population cannot move across the boundary of the domain. And we incorporate a single discrete delay $\tau>0$ in the negative feedback of the predator's density.

The rest of the paper is organized as follows. In Section 2, we give the stability property of the equilibria of model (1). In Section 3, we mainly analyze the distribution of the roots of the characteristic equation and show the occurrence of Hopf bifurcation at the positive equilibrium of model (2) under some conditions. In Section 4, we investigate the stability and direction of bifurcating periodic orbits by using normal form of theorem and the center manifold theorem, corresponding to theorems we also give some numerical simulations.

\section{Equilibria Stability}

In this section, we consider the existence and stability of the equilibria of model (1).

It is easy to verify that model (1) always has three boundary equilibria:

(i) $E_{1}=(0,0)$ (extinction of prey and predator), which is a nodal source point;

(ii) $E_{2}=\left(a_{1} / b, 0\right)$ (extinction of the predator), which is a saddle point;

(iii) $E_{3}=\left(0, a_{2} k_{2} / c_{2}\right)$ (extinction of the prey), which is a stable node when $a_{2} k_{2} / c_{2}>a_{1} k_{1} / c_{1}$.

For the positive equilibria, we have

$$
a_{1}-b H-\frac{c_{1} P}{k_{1}+H}=0, \quad a_{2}-\frac{c_{2} P}{k_{2}+H}=0,
$$

which yields

$$
b c_{2} H^{2}-\left(a_{1} c_{2}-a_{2} c_{1}-b c_{2} k_{1}\right) H+a_{2} c_{1} k_{2}-a_{1} c_{2} k_{1}=0 .
$$

For simplicity, we define

$$
\begin{gathered}
A=b c_{2}, \quad B=a_{1} c_{2}-a_{2} c_{1}-b c_{2} k_{1}, \\
C=a_{2} c_{1} k_{2}-a_{1} c_{2} k_{1},
\end{gathered}
$$

then (4) can be written as

$$
A H^{2}-B H+C=0,
$$

which has two roots given by

$$
h_{+}=\frac{B+\sqrt{B^{2}-4 A C}}{2 A}, \quad h_{-}=\frac{B-\sqrt{B^{2}-4 A C}}{2 A} .
$$

Case 1 ( $C<0$, i.e., $\left.k_{2}<a_{1} c_{2} k_{1} / a_{2} c_{1}\right)$. Model (1) has a unique positive equilibrium $E^{*}=\left(h^{*}, p^{*}\right)=\left(h_{+},\left(a_{2} / c_{2}\right)\left(h_{+}+k_{2}\right)\right)$.

Case $2\left(C=0\right.$, i.e., $\left.k_{2}=a_{1} c_{2} k_{1} / a_{2} c_{1}\right)$.

(i) If $B>0$, that is, $k_{1}<\left(a_{1} c_{2}-a_{2} c_{1}\right) / b c_{2}$, model (1) has a unique positive equilibrium $\bar{E}=(\bar{h}, \bar{p})=\left(h_{+},\left(a_{2}\right)\right.$ $\left.\left.c_{2}\right)\left(h_{+}+k_{2}\right)\right)$;

(ii) If $B \leq 0$, that is, $k_{1} \geq\left(a_{1} c_{2}-a_{2} c_{1}\right) / b c_{2}$, (4) has no positive root; hence model (1) has no positive equilibrium.

Case $3\left(C>0\right.$, i.e., $\left.k_{2}>a_{1} c_{2} k_{1} / a_{2} c_{1}\right)$.

(i) Suppose that $B>0$, that is, $k_{1}<\left(a_{1} c_{2}-a_{2} c_{1}\right) / b c_{2}$, then

(i1) if $B^{2}-4 A C>0$, that is, $k_{2}<\left(a_{2} c_{1}-a_{1} c_{2}-\right.$ $\left.b c_{2} k_{1}\right)^{2} / 4 a_{2} b c_{1} c_{2}+k_{1}$, model (1) has two positive equilibria $E_{+}=\left(h_{+}, p_{+}\right)=\left(h_{+},\left(a_{2} / c_{2}\right)\left(h_{+}+k_{2}\right)\right)$ and $E_{-}=\left(h_{-}, p_{-}\right)=\left(h_{-},\left(a_{2} / c_{2}\right)\left(h_{-}+k_{2}\right)\right)$;

(i2) if $B^{2}-4 A C=0$, that is, $k_{2}=\left(a_{2} c_{1}-a_{1} c_{2}-\right.$ $\left.b c_{2} k_{1}\right)^{2} / 4 a_{2} b c_{1} c_{2}+k_{1}$, (4) has a unique positive root of multiplicity 2 given by $h_{e}=B / 2 A=$ $h_{+}=h_{-}$, then model (1) has a unique positive equilibrium $E_{e}=\left(h_{e}, p_{e}\right)=\left(h_{e},\left(a_{2} / c_{2}\right)\left(h_{e}+k_{2}\right)\right)$;

(i3) if $B^{2}-4 A C=0$, that is, $k_{2}>\left(a_{2} c_{1}-a_{1} c_{2}-\right.$ $\left.b c_{2} k_{1}\right)^{2} / 4 a_{2} b c_{1} c_{2}+k_{1}$, (4) has no positive root; hence model (1) has no positive equilibrium;

(ii) if $B<0$, that is, $k_{1}>\left(a_{1} c_{2}-a_{2} c_{1}\right) / b c_{2}$, (4) has no positive root; hence model (1) has no positive equilibrium.

We show the bifurcation diagram to display the distribute of the positive roots; in Figure 1, the whole region has been divided into six parts; the number indicates the number of positive equilibria.

Next, we analyze the stability of these positive equilibria. Let $E=(h, p)$ be arbitrary positive equilibrium, and the Jacobian matrix for $E=(h, p)$ is given by

$$
J(E)=\left(\begin{array}{cc}
-b h+\frac{c_{1} h p}{\left(h+k_{1}\right)^{2}} & \frac{-c_{1} h}{h+k_{1}} \\
\frac{a_{2}^{2}}{c_{2}} & -a_{2}
\end{array}\right) .
$$




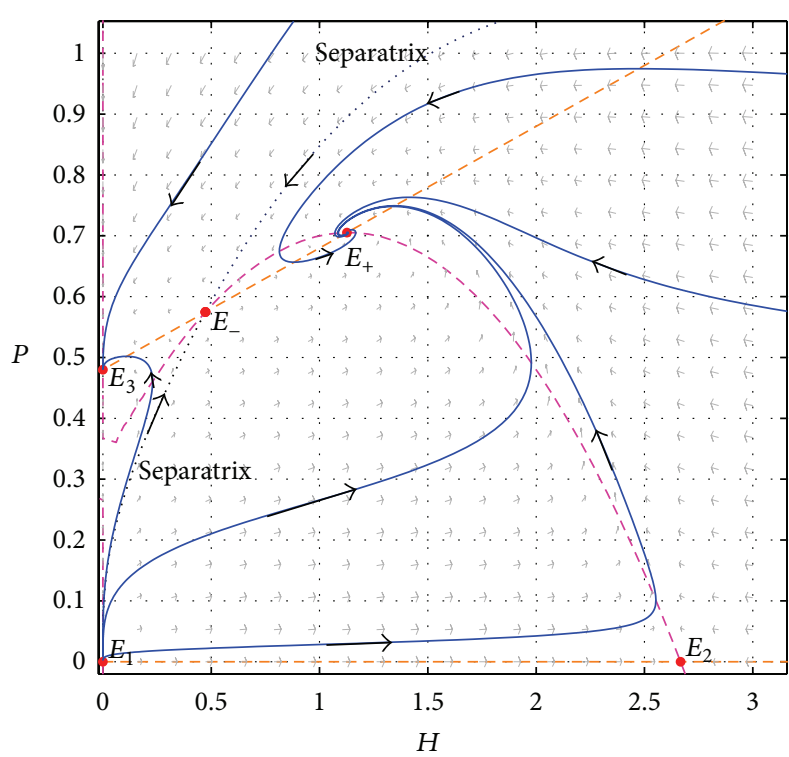

FIGURE 2: Phase portraits of model (1) with the parameters $a_{1}=2$, $a_{2}=0.5, b=1, c_{1}=c_{2}=2, k_{1}=0.4$, and $k_{2}=2.1$. In this case, $E_{1}=(0,0)$ is a nodal source point; $E_{2}=(2,0)$ is a saddle point; $E_{3}=$ $(0,0.525)$ is a nodal sink point, which is locally asymptotically stable; $E_{+}=(0.7791,0.7197)$ is a spiral sink, which is locally asymptotically stable; and $E_{-}=(0.3208,0.6052)$ is a saddle point. There exists a separatrix curve determined by the stable manifold of $E_{-}$. The dashed curve is the $H$-nullcline $H\left(a_{1}-b H\right)-c_{1} H P /\left(k_{1}+H\right)$, and the dotted curve is the $P$-nullcline $P\left(a_{2}-c_{2} P /\left(k_{2}+H\right)\right)$.

The corresponding characteristic equation is

$$
\lambda^{2}-\operatorname{tr}(J(E)) \lambda+\operatorname{det}(J(E))=0,
$$

where

$$
\begin{aligned}
\operatorname{tr}(J(E)) & =\frac{c_{1} h p}{\left(h+k_{1}\right)^{2}}-a_{2}-b h \\
& =\frac{h\left(a_{1}-a_{2}-2 b h\right)-\left(a_{2}+b h\right) k_{1}}{h+k_{1}}, \\
\operatorname{det}(J(E)) & =\frac{a_{2} h\left(a_{2} c_{1}-a_{1} c_{2}+b c_{2} k_{1}+2 b c_{2} h\right)}{c_{2}\left(h+k_{1}\right)} .
\end{aligned}
$$

And the sign of $\operatorname{det}(J(E))$ is determined by

$$
F_{1}(h)=a_{2} c_{1}-a_{1} c_{2}+b c_{2} k_{1}+2 b c_{2} h=2 A h-B .
$$

Thus,

$$
\begin{gathered}
F_{1}\left(h^{*}\right)=2 A h^{*}-B=\sqrt{B^{2}-4 A C}>0, \\
F_{1}(\bar{h})=2 A \bar{h}-B=B>0, \\
F_{1}\left(h_{+}\right)=2 A h_{+}-B=\sqrt{B^{2}-4 A C}>0 ; \\
F_{1}\left(h_{-}\right)=2 A h_{-}-B=-\sqrt{B^{2}-4 A C}<0, \\
F_{1}\left(h_{e}\right)=2 A h_{e}-B=0 .
\end{gathered}
$$

Hence, $\operatorname{det}\left(J\left(E^{*}\right)\right)>0, \operatorname{det}(J(\bar{E}))>0, \operatorname{det}\left(J\left(E_{+}\right)\right)>0$, $\operatorname{det}\left(J\left(E_{-}\right)\right)<0$, and $\operatorname{det}\left(J\left(E_{e}\right)\right)=0$. Obviously, the positive equilibrium $E_{-}$is a saddle point.

In the following, we study the stability of other positive equilibria. The sign of $\operatorname{tr}(J(E))$ is determined by

$$
G_{1}(h)=h\left(a_{1}-a_{2}-2 b h\right)-\left(a_{2}+b h\right) k_{1} .
$$

Then we can get

$$
\begin{gathered}
G_{1}\left(h^{*}\right)=\frac{h^{*} a_{2}\left(c_{1}-c_{2}\right)}{c_{2}}-\frac{h^{*} \sqrt{B^{2}-4 A C}}{c_{2}}-a_{2} k_{1}, \\
G_{1}(\bar{h})=\frac{\bar{h} a_{2}\left(c_{1}-c_{2}\right)}{c_{2}}-\frac{\bar{h} B}{c_{2}}-a_{2} k_{1}, \\
G_{1}\left(h_{+}\right)=\frac{h_{+} a_{2}\left(c_{1}-c_{2}\right)}{c_{2}}-\frac{h_{+} \sqrt{B^{2}-4 A C}}{c_{2}}-a_{2} k_{1}, \\
G_{1}\left(h_{e}\right)=\frac{h_{e} a_{2}\left(c_{1}-c_{2}\right)}{c_{2}}-a_{2} k_{1} .
\end{gathered}
$$

Hence, if $c_{1} \leq c_{2}$, then $\operatorname{tr}\left(J\left(E^{*}\right)\right)<0, \operatorname{tr}(J(\bar{E}))<0$, and $\operatorname{tr}\left(J\left(E_{+}\right)\right)<0$ are true. Summarizing the above, we can obtain the following theorem.

Theorem 1. For model (1),

(i) if $k_{2}<a_{1} c_{2} k_{1} / a_{2} c_{1}$ holds, the unique positive equilibrium $E^{*}$ is locally asymptotically stable for $c_{1} \leq c_{2}$;

(ii) if $k_{2}=a_{1} c_{2} k_{1} / a_{2} c_{1}$ and $k_{1}<\left(a_{1} c_{2}-a_{2} c_{1}\right) / b c_{2}$ hold, the unique positive equilibrium $\bar{E}$ is locally asymptotically stable for $c_{1} \leq c_{2}$;

(iii) if $a_{1} c_{2} k_{1} / a_{2} c_{1}<k_{2}<\left(a_{2} c_{1}-a_{1} c_{2}-b c_{2} k_{1}\right)^{2} / 4 a_{2} b c_{1} c_{2}+$ $k_{1}$ and $k_{1}<\left(a_{1} c_{2}-a_{2} c_{1}\right) / b c_{2}$ hold, model (1) has two positive equilibria, the positive equilibrium $E_{+}$is locally asymptotically stable for $c_{1} \leq c_{2}$, and $E_{-}$is a saddle point.

Figure 2 shows the dynamics of model (1). In this case, $E_{1}$ is a nodal source point; $E_{2}$ is a saddle point; $E_{3}$ is a nodal sink point, which is locally asymptotically stable; $E_{+}$ is locally asymptotically stable; $E_{-}$is a saddle point. There exists a separatrix curve determined by the stable manifold of $E_{-}$, which divides the behavior of trajectories; that is, the stable manifold of saddle $E_{-}$splits the feasible region into two parts such that orbits initiating inside tend to the positive equilibrium $E_{+}$, while orbits initiating outside tend to $E_{3}$ except for the stable manifolds of $E_{-}$. This means that, in this situation, the trajectories of the model can have different behavior strongly depending on the initial conditions.

Theorem 2. For model (1), if the unique positive equilibrium $E_{e}=\left(h_{e},\left(a_{2} / c_{2}\right)\left(h_{e}+k_{2}\right)\right)$ exists, and $k_{1}=\left(a_{1} c_{2}-a_{2} c_{1}\right)\left(c_{1}-\right.$ $\left.c_{2}\right) / b c_{2}\left(c_{1}+c_{2}\right)$, then it is a cusp of codimension 2 . 
Proof. The Jacobian matrix at $E_{e}=\left(h_{e}, p_{e}\right)$ is

$$
\begin{aligned}
& J_{E_{e}} \\
& =\left(\begin{array}{cc}
-\frac{a_{2} c_{1}\left(a_{2} c_{1}-a_{1} c_{2}+b c_{2} k_{1}\right)}{c_{2}\left(b c_{2} k_{1}-a_{2} c_{1}+a_{1} c_{2}\right)} & \frac{c_{1}\left(a_{2} c_{1}-a_{1} c_{2}+b c_{2} k_{1}\right)}{b c_{2} k_{1}-a_{2} c_{1}+a_{1} c_{2}} \\
\frac{a_{2}^{2}}{c_{2}} & -a_{2}
\end{array}\right),
\end{aligned}
$$

we have know that $\operatorname{det}\left(J\left(E_{e}\right)\right)=0$. Moreover, $\operatorname{tr}\left(J\left(E_{e}\right)\right)=0$, if and only if

$$
k_{1}=\frac{\left(a_{1} c_{2}-a_{2} c_{1}\right)\left(c_{1}-c_{2}\right)}{b c_{2}\left(c_{1}+c_{2}\right)} .
$$

Then

$$
J_{E_{e}}=\left(\begin{array}{cc}
a_{2} & -c_{2} \\
\frac{a_{2}^{2}}{c_{2}} & -a_{2}
\end{array}\right)=a_{2}\left(\begin{array}{cc}
1 & -\frac{c_{2}}{a_{2}} \\
\frac{a_{2}}{c_{2}} & -1
\end{array}\right) \text {, }
$$

and the associate Jordan matrix is

$$
\widehat{J}=\left(\begin{array}{cc}
0 & \frac{c_{1}\left(a_{2} c_{1}-a_{1} c_{2}+b c_{2} k_{1}\right)}{b c_{2} k_{1}-a_{2} c_{1}+a_{1} c_{2}} \\
0 & 0
\end{array}\right) .
$$

Hence, following $[15,16]$, we know that the unique positive equilibrium $E_{e}$ is a cusp of codimension 2 .

\section{Stability and Hopf Bifurcation Analysis in Delayed Reaction-Diffusion Model (2)}

According to the previous section, for model (1), we know that $E_{1}, E_{2}$, and $E_{3}$ are unstable and $E_{-}$is a saddle point, and note that a solution of the model (1) is also a solution of the model (2), so they are also unstable for model (2). In the following, we will focus on the dynamics of the positive equilibria of model (2). As an example, we only give the proof of the unique positive equilibrium $E^{*}$ of model (2).

Introducing small perturbations $\widetilde{H}=H-h^{*}$, and $\widetilde{P}=$ $P-p^{*}$ and dropping the hats for simplicity of notation, then we have

$$
\begin{aligned}
\frac{\partial H}{\partial t}= & \left(H+h^{*}\right)\left(a_{1}-b\left(H+h^{*}\right)\right) \\
& -\frac{c_{1}\left(H+h^{*}\right)\left(P(t-\tau)+p^{*}\right)}{k_{1}+H+h^{*}} \\
& +D_{1} \Delta H, \quad x \in \Omega, t>0, \\
\frac{\partial P}{\partial t}= & \left(P+p^{*}\right)\left(a_{2}-\frac{c_{2}\left(P+p^{*}\right)}{k_{2}+H+h^{*}}\right) \\
& +D_{2} \Delta P, \quad x \in \Omega, t>0, \\
\frac{\partial H}{\partial \mathbf{n}} & =\frac{\partial P}{\partial \mathbf{n}}=0, \quad x \in \partial \Omega, t>0 .
\end{aligned}
$$

$$
\begin{aligned}
& \text { Denote } \\
& X=\left\{H, P \in W^{2,2}(\Omega): \frac{\partial H}{\partial \mathbf{n}}=\frac{\partial P}{\partial \mathbf{n}}=0, x \in \partial \Omega\right\} .
\end{aligned}
$$

In the abstract space $C([-\tau, 0], X)$, model (19) can be regarded as the following abstract functional differential equation.

$$
\frac{\partial U}{\partial t}=D \Delta U+L\left(U_{t}\right)+F\left(U_{t}\right)
$$

where $U=(H, P)^{T}, U_{t}=U(t+\theta), \theta \in[-\tau, 0], D=\left(\begin{array}{cc}D_{1} & \\ & D_{2}\end{array}\right)$, $\operatorname{dom}(\Delta) \subset X$, and $L: C([-\tau, 0], X) \mapsto X, F: C([-\tau, 0], X) \mapsto$ $X$ are given by

$L(\phi)$

$$
=\left(\begin{array}{c}
\left.-b h^{*}+\frac{c_{1} h^{*} p^{*}}{\left(h^{*}+k_{1}\right)^{2}}\right) \phi_{1}(0)+\frac{-c_{1} h^{*}}{h^{*}+k_{1}} \phi_{2}(-\tau) \\
\frac{a_{2}^{2}}{c_{2}} \phi_{1}(0)-a_{2} \phi_{2}(0)
\end{array}\right),
$$

$F(\phi)$

$$
=\left(\begin{array}{c}
\left(\frac{c_{1} k_{1} p^{*}}{\left(k_{1}+h^{*}\right)^{3}}-b\right) \phi_{1}^{2}(0)-\frac{c_{1} k_{1}}{\left(k_{1}+h^{*}\right)^{2}} \phi_{1}(0) \phi_{2}(-\tau) \\
-\frac{c_{2} p^{* 2}}{\left(k_{2}+h^{*}\right)^{3}} \phi_{1}^{2}(0)+\frac{2 c_{2} p^{*}}{\left(k_{2}+h^{*}\right)^{2}} \phi_{1}(0) \phi_{2}(0)-\frac{c_{2}}{k_{2}+h^{*}} \phi_{2}^{2}(0)
\end{array}\right)
$$

here $\phi=\left(\phi_{1}, \phi_{2}\right)^{T}=U_{t} \in C([-\tau, 0], X)$. Then the linearization of model (19) near $\left(h^{*}, p^{*}\right)$ is

$$
\frac{\partial U}{\partial t}=D \Delta U+L\left(U_{t}\right)
$$

Following [17], we obtain that the characteristic equation for liner model (23) is

$$
\lambda y-D \Delta y-L\left(e^{\lambda \cdot} y\right)=0, \quad y \in \operatorname{dom}(\Delta) \subset X, \quad y \neq 0 .
$$

It is well known that the eigenvalue problem

$$
\begin{gathered}
-\Delta \psi=\mu \psi, \quad x \in \Omega, \\
\frac{\partial \psi}{\partial \mathbf{n}}=0, \quad x \in \partial \Omega,
\end{gathered}
$$

has eigenvalues $\mu_{n}=\left\{\mu_{n}^{i}=-D_{i} n^{2}, i=1,2\right.$, and $\left.n=0,1,2, \ldots\right\}$, with the corresponding eigenfunctions $\psi_{n}(x)=\cos n x \quad(n \in$ $\mathbb{N}=\{0,1,2, \ldots\})$.

Substituting $y=\sum_{n=0}^{\infty} \cos n x\left(y_{1 n}, y_{2 n}\right)^{T}$ into characteristic equation (24), we obtain

$$
\begin{gathered}
\left(\begin{array}{cc}
-b h^{*}+\frac{c_{1} h^{*} p^{*}}{\left(h^{*}+k_{1}\right)^{2}}-D_{1} n^{2} & \frac{-c_{1} h^{*}}{h^{*}+k_{1}} e^{-\lambda \tau} \\
\frac{a_{2}^{2}}{c_{2}} & -a_{2}-D_{2} n^{2}
\end{array}\right)\left(\begin{array}{l}
y_{1 n} \\
y_{2 n}
\end{array}\right) \\
=\lambda\left(\begin{array}{l}
y_{1 n} \\
y_{2 n}
\end{array}\right) .
\end{gathered}
$$


Therefore the characteristic equation (24) is equivalent to

$$
\lambda^{2}+A_{n} \lambda+B_{n} e^{-\lambda \tau}+C_{n}=0, \quad n \in \mathbb{N}
$$

where

$$
\begin{aligned}
A_{n}= & \left(D_{1}+D_{2}\right) n^{2}+a_{2}+b h^{*}-\frac{c_{1} h^{*} p^{*}}{\left(h^{*}+k_{1}\right)^{2}}, \\
B_{n}= & \frac{a_{2}^{2} c_{1} h^{*}}{c_{2}\left(h^{*}+k_{1}\right)}, \\
C_{n}= & D_{1} D_{2} n^{4}+\left(D_{1}+D_{2}+a_{2}+b h^{*}-\frac{c_{1} h^{*} p^{*}}{\left(h^{*}+k_{1}\right)^{2}}\right) n^{2} \\
& +a_{2} b h^{*}-\frac{a_{2} c_{1} h^{*} p^{*}}{\left(h^{*}+k_{1}\right)^{2}} .
\end{aligned}
$$

The stability of the positive equilibrium $E^{*}$ can be determined by the distribution of the roots of (27); that is, the equilibrium $E^{*}$ is locally asymptotically stable if all the roots of (27) have negative real parts. From the result of [18], the sum of the multiplicities of the roots of (27) in the open right half plane changes only if a root appears on or crosses the imaginary axis. It can be verified that $\lambda=0$ is not a root of (27) for $n \in \mathbb{N}$.

Theorem 3. If $k_{2}<\min \left\{a_{1} c_{2} k_{1} / a_{2} c_{1}, b c_{2}\left(h^{*}+k_{1}\right)^{2} / a_{2} c_{1}-\right.$ $\left.2 h^{*}-k_{1}\right\}$ holds, then the unique positive equilibrium $E^{*}$ of model (2) is locally asymptotically stable.

Proof. Let $\pm i \omega(\omega>0)$ be a pair of roots of (27); substituting $i \omega$ into (27), then we have

$$
-\omega^{2}+i A_{n} \omega+B_{n} e^{-i \omega \tau}+C_{n}=0 .
$$

Separating the real part from image part, we have

$$
\begin{gathered}
-\omega^{2}+B_{n} \cos \omega \tau+C_{n}=0, \\
A_{n} \omega-B_{n} \sin \omega \tau=0,
\end{gathered}
$$

then

$$
\omega^{4}+\left(A_{n}^{2}-2 C_{n}\right) \omega^{2}+C_{n}^{2}-B_{n}^{2}=0
$$

where

$$
\begin{aligned}
A_{n}^{2}-2 C_{n}= & \left(b h^{*}-\frac{c_{1} h^{*} p^{*}}{\left(h^{*}+k_{1}\right)^{2}}+D_{1} n^{2}\right)^{2} \\
& +\left(a_{2}+D_{2} n^{2}\right)^{2}>0 \\
C_{n}^{2}-B_{n}^{2}= & D_{1} D_{2} n^{4} \\
& +\left(D_{1}+D_{2}+a_{2}+b h^{*}-\frac{c_{1} h^{*} p^{*}}{\left(h^{*}+k_{1}\right)^{2}}\right) n^{2} \\
& -\frac{a_{2} c_{1} h^{*} p^{*}}{\left(h^{*}+k_{1}\right)^{2}}-\frac{a_{2}^{4} c_{1}^{2}\left(h^{*}\right)^{2}}{c_{2}^{2}\left(h^{*}+k_{1}\right)^{2}}+a_{2} b h^{*} .
\end{aligned}
$$

Obviously, if $k_{2}<b c_{2}\left(h^{*}+k_{1}\right)^{2} / a_{2} c_{1}-2 h^{*}-k_{1}, C_{n}^{2}-B_{n}^{2}>0$ is true. Thus (31) has no positive roots for all $n \in \mathbb{N}$. Hence, all the roots of (27) have negative real part. This completes the proof.

If there exists an integer $n_{0} \in \mathbb{N}$ such that for $0 \leq n \leq n_{0}$, $C_{n}^{2}-B_{n}^{2}<0$, then (31) has a unique positive real root

$$
\omega_{0}^{n}=\sqrt{\frac{-\left(A_{n}^{2}-2 C_{n}\right)+\sqrt{\left(A_{n}^{2}-2 C_{n}\right)^{2}-4\left(C_{n}^{2}-B_{n}^{2}\right)}}{2}},
$$

and (27) has a pair of pure imaginary roots $\pm i \omega_{0}^{n}$, and

$$
\tau_{n}^{j}=\tau_{n}^{0}+\frac{2 \pi j}{\omega_{n}}, \quad j=0,1,2, \ldots, 0 \leq n \leq n_{0}, n_{0} \in \mathbb{N}
$$

where $\tau_{n}^{0}=\arccos \left(\left(\omega_{0}^{n 2}-C_{n}\right) / B_{n}\right) / \omega_{0}^{n}$.

Let $\lambda(\tau)=\gamma(\tau)+i \omega(\tau)$ be the root of (24), where $\gamma\left(\tau_{n}^{j}\right)=$ 0 and $\omega\left(\tau_{n}^{j}\right)=\omega_{0}^{n}$ when $\tau$ is close to $\tau_{n}^{j}$. Then we have the following transversality condition.

Lemma 4. For $0 \leq n \leq n_{0}\left(n_{0} \in \mathbb{N}\right)$, if

$$
\text { (H1) } \begin{array}{ll}
D_{1} D_{2} n^{4}+\left(D_{1}+D_{2}+a_{2}+b h^{*}-c_{1} h^{*} p^{*} /\left(h^{*}+k_{1}\right)^{2}\right) n^{2}< \\
\\
a_{2} c_{1} h^{*} p^{*} /\left(h^{*}+k_{1}\right)^{2}+a_{2}^{4} c_{1}^{2}\left(h^{*}\right)^{2} / c_{2}^{2}\left(h^{*}+k_{1}\right)^{2}-a_{2} b h^{*} \\
\text { holds, then }\left.(d \gamma / d \tau)\right|_{\tau=\tau_{n}^{j}}>0 \text { for } j=0,1,2, \ldots
\end{array}
$$

From this transversality condition, we know that when $\tau$ passes through these critical values $\tau_{n}^{j}$, the sum of the multiplicities of the roots of (27) in the open right half plane will increases at least two.

Summarizing the above results, we can obtain the following theorem.

Theorem 5. For $0 \leq n \leq n_{0}\left(n_{0} \in \mathbb{N}\right)$ if (H1) holds, the following statements are true:

(i) if $\tau \in\left[0, \tau_{n}^{0}\right)$, then the equilibrium point $E^{*}$ is locally asymptotically stable;

(ii) if $\tau>\tau_{n}^{0}$, then the equilibrium $E^{*}$ is unstable;

(iii) $\tau=\tau_{n}^{j}(j=0,1,2, \ldots)$ are Hopf bifurcation values of model (2).

\section{Direction and Stability of Spatial Hopf Bifurcation}

In the previous section, we have obtained the conditions under which model (2) undergoes a Hopf bifurcation at the the equilibrium point $E^{*}$ when $\tau$ crosses though the critical value $\tau_{n}^{j}\left(0 \leq n \leq n_{0}, n_{0} \in \mathbb{N}, j=0,1,2, \ldots\right)$. In this section, we will study the direction of the Hopf bifurcation and the stability of the bifurcating periodic solutions by employing the center manifold theorem and normal form method $[17,19]$ for partial differential equations with delay. 
Then we compute the direction and stability of the Hopf bifurcation when $\tilde{\tau}=\tau_{n}^{j}$ for fixed $j \in\{0,1,2, \ldots\}$.

Define

$$
\begin{gathered}
\mu=-\frac{\operatorname{Re}\left(c_{1}(0)\right)}{\operatorname{Re}\left(\lambda^{\prime}(\tilde{\tau})\right)}, \\
\beta_{2}=2 \operatorname{Re}\left(c_{1}(0)\right), \\
T_{2}=-\frac{\operatorname{Im}\left(c_{1}(0)\right)+\mu_{2} \operatorname{Im}\left(\lambda^{\prime}(\widetilde{\tau})\right)}{\omega_{0}^{n} \widetilde{\tau}},
\end{gathered}
$$

where $c_{1}(0)$ is defined in the appendix. Then we can get the following theorem.

Theorem 6. For model (19), if (H1) holds, we have the following:

(i) $\mu$ determines the direction of the Hopf bifurcation: if $\mu>0(\mu<0)$, then the bifurcating periodic solutions exist for $\tau>\tilde{\tau}(\tau<\tilde{\tau})$;

(ii) $\beta_{2}$ determines the stability of bifurcating periodic solutions: the bifurcating periodic solutions are orbitally asymptotically stable (unstable) if $\beta_{2}<0\left(\beta_{2}>0\right)$;

(iii) $T_{2}$ determines the period of the bifurcating periodic solutions: the period increases (decreases) if $T_{2}>0$ $\left(T_{2}<0\right)$.

The proof is deferred to the appendix.

\section{Conclusions and Remarks}

In this paper, we have considered a modified version of Leslie-Gower model with Holling-type II functional and delayed diffusive predator-prey model under homogeneous Neumann boundary conditions. The value of this study lies in two folds. First, it presents local asymptotic stability of the equilibria of model with and without delay and the existence of Hopf bifurcation, which indicates that the dynamics induced by time delay are rich and complex. Second, it give the analysis of direction and stability of spatial Hopf bifurcation, from which one can find that small sufficiently delays cannot change the stability of the positive equilibrium and large delays cannot only destabilize the positive equilibrium but also induce oscillatory behaviors near the positive equilibrium.

In the following, we give some numerical examples to illustrate the dynamical behaviors of model (2). In Figure 3, $\tau=2<\tau_{0}^{0}=3.435144529$, the unique positive equilibrium $E^{*}=(2,1.5)$ remains the stability; the population of the predator and the prey will tend to a steady state. However, in Figure 4, $\tau=4>\tau_{0}^{0}$, the positive equilibrium $E^{*}$ losses its stability and Hopf bifurcation occurs, which means that a family of stable periodic solutions bifurcate from $E^{*}$ and the system goes into oscillations; it means that the predator coexists with the prey with oscillatory behaviors.

Our results show that time-delay can make a stable equilibrium to become unstable and induce Hopf bifurcation and the system goes into oscillations; that's to say, the dynamical behaviors of the delay reaction-diffusion equations are much more complex and rich than reaction-diffusion equations.

\section{Appendix}

\section{A. The Proof of Theorem 6}

Setting $\tau=\tilde{\tau}+\alpha$, then $\alpha=0$ is the Hopf bifurcation of model (19). Let $\widetilde{H}(t, x)=H(\tau t, x), \widetilde{P}(t, x)=P(\tau t, x)$, and drop the tilde for the sake of simplicity, then (19) can be transformed into

$$
\begin{aligned}
\frac{\partial H}{\partial t}= & \tau\left(H+h^{*}\right)\left(a_{1}-b\left(H+h^{*}\right)\right) \\
& -\frac{c_{1}\left(H+h^{*}\right)\left(P(t-1)+p^{*}\right)}{k_{1}+H+h^{*}} \\
& +D_{1} \Delta H, \quad x \in \Omega, t>0, \\
\frac{\partial P}{\partial t}= & \tau\left(P+p^{*}\right)\left(a_{2}-\frac{c_{2}\left(P+p^{*}\right)}{k_{2}+H+h^{*}}\right) \\
& +D_{2} \Delta P, \quad x \in \Omega, t>0, \\
\frac{\partial H}{\partial \mathbf{n}}= & \frac{\partial P}{\partial \mathbf{n}}=0, \quad x \in \partial \Omega, t>0 .
\end{aligned}
$$

And the abstract functional differential equation can also be written in the form

$$
\frac{d U}{d t}=\tilde{\tau} D \Delta U+\tilde{\tau} L\left(U_{t}\right)+G\left(U_{t}, \alpha\right),
$$

where

$L(\phi)$

$$
\begin{gathered}
=\left(\begin{array}{c}
\left(-b h^{*}+\frac{c_{1} h^{*} p^{*}}{\left(h^{*}+k_{1}\right)^{2}}\right) \phi_{1}(0)-\frac{c_{1} h^{*}}{h^{*}+k_{1}} \phi_{2}(-1) \\
\frac{a_{2}^{2}}{c_{2}} \phi_{1}(0)-a_{2} \phi_{2}(0)
\end{array}\right), \\
G(\phi, \alpha)=\alpha D \Delta \phi+\alpha L(\phi)+(\tilde{\tau}+\alpha) F(\phi),
\end{gathered}
$$

$F(\phi)$

$$
=\left(\begin{array}{c}
\left(\frac{c_{1} k_{1} p^{*}}{\left(k_{1}+h^{*}\right)^{3}}-b\right) \phi_{1}^{2}(0)-\frac{c_{1} k_{1}}{\left(k_{1}+h^{*}\right)^{2}} \phi_{1}(0) \phi_{2}(-1) \\
-\frac{c_{2} p^{* 2}}{\left(k_{2}+h^{*}\right)^{3}} \phi_{1}^{2}(0)+\frac{2 c_{2} p^{*}}{\left(k_{2}+h^{*}\right)^{2}} \phi_{1}(0) \phi_{2}(0)-\frac{c_{2}}{k_{2}+h^{*}} \phi_{2}^{2}(0)
\end{array}\right),
$$

for $\phi=\left(\phi_{1}, \phi_{2}\right)=U_{t} \in C([-1,0], X)$.

From Section 2, we know that $\pm i \omega_{0}^{n} \tilde{\tau}$ are a pair of simple purely imaginary eigenvalues of the liner system

$$
\frac{d U}{d t}=\tilde{\tau} L\left(U_{t}\right)+\widetilde{\tau} D \Delta U
$$

where $U(t)=(H(t), P(t))^{T} \in R^{2}$, and $U_{t}(\theta)$ is defined by $U_{t}(\theta)=U(t+\theta)$. 


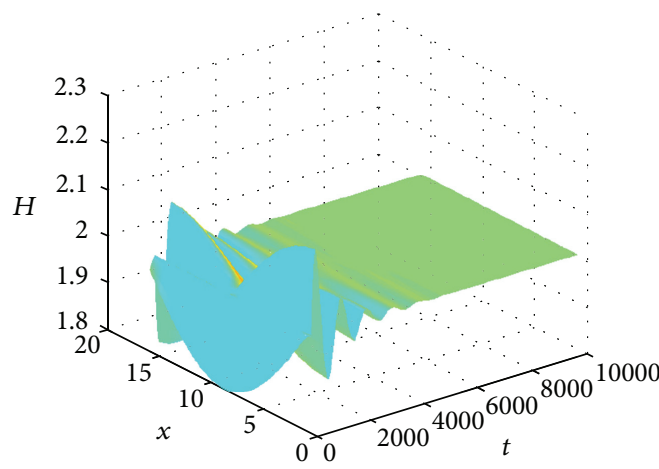

(a)

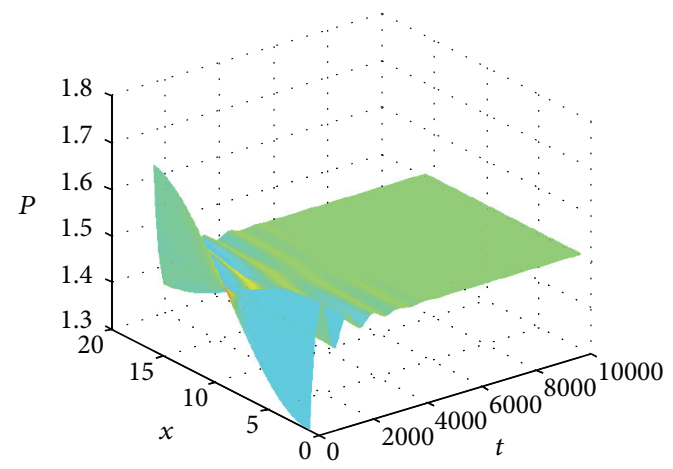

(b)

Figure 3: The solutions of model (2) tends to aperiodically oscillatory orbit. The parameters are taken as $D_{1}=0.01, D_{2}=0.1, a_{1}=2, a_{2}=1$, $b=0.5, c_{1}=c_{2}=2, k_{1}=k_{2}=1$, and the initial values $H(x, t)=2+0.1 t \sin x, P(x, t)=1.5+0.1 t \cos x . t \in[-2,0], x \in[0, \pi]$. In this case, $\tau=4>\tau_{0}^{0}$.

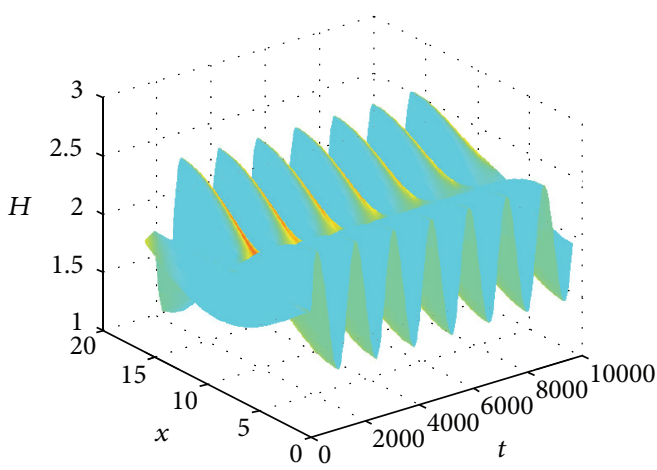

(a)

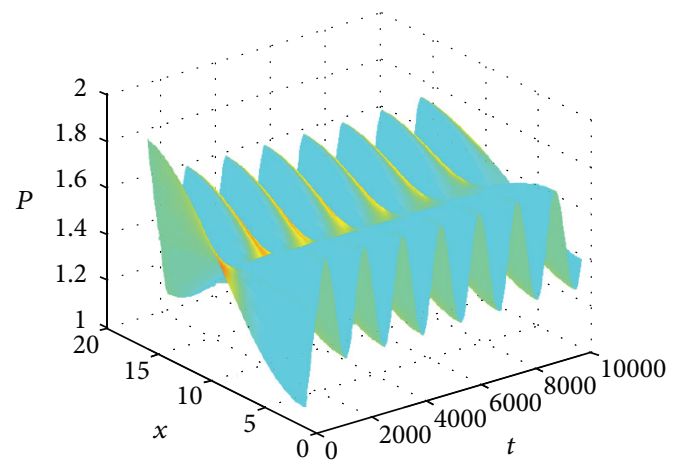

(b)

FIGURE 4: The solutions of model (2) tends to aperiodically oscillatory orbit. The parameters are taken as $D_{1}=0.01, D_{2}=0.1, a_{1}=2, a_{2}=1$, $b=0.5, c_{1}=c_{2}=2, k_{1}=k_{2}=1$, and the initial values $H(x, t)=2+0.1 t \sin x, P(x, t)=1.5+0.1 t \cos x . t \in[-4,0], x \in[0, \pi]$. In this case, $\tau=4>\tau_{0}^{0}$.

By using the Riesz representation theorem [20], we have a function $\eta(\theta, \alpha)$ of bounded variation for $\theta \in[-1,0]$ such that

$$
\begin{array}{r}
L(\phi)+D \Delta \phi(0)=\int_{-1}^{0} d \eta(\theta, \alpha) \phi(\theta), \\
\text { for } \phi(\theta) \in C\left([-1,0], R^{2}\right) .
\end{array}
$$

Because in this paper, we discuss the existence of the Hopf bifurcation when $\tau=\tau_{0}^{0}$, that is $n=0$; here we choose

$$
\begin{aligned}
& \eta(\theta, \alpha)=\left(\begin{array}{cc}
-b h^{*}+\frac{c_{1} h^{*} p^{*}}{\left(h^{*}+k_{1}\right)^{2}} & 0 \\
\frac{a_{2}^{2}}{c_{2}} & -a_{2}
\end{array}\right) \delta(\theta) \\
& +\left(\begin{array}{cc}
0 & \frac{-c_{1} h^{*}}{h^{*}+k_{1}} \\
0 & 0
\end{array}\right) \delta(\theta+1),
\end{aligned}
$$

where $\delta$ is the Dirac delta function. For $\phi(\theta) \in C^{1}\left([-1,0], R^{2}\right)$, define $A(\alpha)$ as

$$
A(\alpha) \phi= \begin{cases}\frac{d \phi(\theta)}{d \theta}, & \theta \in[-1,0), \\ \int_{-1}^{0} d \eta(\theta, \alpha) \phi(\theta), & \theta=0,\end{cases}
$$

and for $\psi=\left(\psi_{1}, \psi_{2}\right) \in C^{1}\left([0,1],\left(R^{2}\right)^{*}\right)$, define

$$
A^{*}(\psi(s))= \begin{cases}-\frac{d \psi(s)}{d s}, & s \in[-1,0), \\ \int_{-1}^{0} \psi(-\xi) d \eta(\theta, 0), & s=0 .\end{cases}
$$


Then $A(0)$ and $A^{*}$ are adjoint operators under the bilinear form

$$
\begin{aligned}
& (\psi(s), \phi(\theta)) \\
& \quad=\bar{\psi}(0) \phi(0)-\int_{-1}^{0} \int_{0}^{\theta} \bar{\psi}(\xi-\theta) d \eta(\theta, \alpha) \phi(\xi) d \xi .
\end{aligned}
$$

We know that $\pm i \omega_{0}^{n}$ are eigenvalues of $A(0)$. Since $A(0)$ and $A^{*}(0)$ are two adjoint operators, then $\pm i \omega_{0}^{n}$ are also eigenvalue of $A^{*}$; we shall first try to obtain eigenvector of $A(0)$ and $A^{*}$ corresponding to the eigenvalues $i \omega_{0}^{n}$ and $-i \omega_{0}^{n}$, respectively. Let $q(\theta)=(1, \rho)^{T} e^{i \omega_{0}^{n} \theta}$ be the eigenvector of $A(0)$ corresponding to the eigenvalue $i \omega_{0}^{n}$. Then by definition of eigenvector we have $A(0) q(\theta)=q(\theta) i \omega_{0}^{n}$. Therefore, from (A.5), (A.6), and definition of $A(0)$ we get

$$
\left(\begin{array}{cc}
-b h^{*}+\frac{c_{1} h^{*} p^{*}}{\left(h^{*}+k_{1}\right)^{2}}-i \omega_{0}^{n} & \frac{-c_{1} h^{*}}{h^{*}+k_{1}} e^{-i \omega_{0}^{n} \tau} \\
\frac{a_{2}^{2}}{c_{2}} & -a_{2}-i \omega_{0}^{n}
\end{array}\right)\left(\begin{array}{l}
1 \\
\rho
\end{array}\right)=\left(\begin{array}{l}
0 \\
0
\end{array}\right),
$$

we choose $\rho=a_{2}^{2} / c_{2}\left(a_{2}+i \omega_{0}^{n}\right)$, and then we get $q(\theta)$. On the other hand, $q^{*}(s)=M(1, \gamma) e^{i \omega_{0}^{n} s}$ is the eigenvector of $A^{*}$ corresponding to the eigenvalue $-i \omega_{0}^{n}$. From the definition of $A^{*}$, we have

$$
\left(\begin{array}{cc}
-b h^{*}+\frac{c_{1} h^{*} p^{*}}{\left(h^{*}+k_{1}\right)^{2}}+i \omega_{0}^{n} & \frac{a_{2}^{2}}{c_{2}} \\
\frac{-c_{1} h^{*}}{h^{*}+k_{1}} e^{-i \omega_{0}^{n} \tau} & -a_{2}+i \omega_{0}^{n}
\end{array}\right)\left(\begin{array}{c}
M \\
M \gamma
\end{array}\right)=\left(\begin{array}{l}
0 \\
0
\end{array}\right),
$$

where

$$
\gamma=\left(b h^{*}-\frac{c_{1} h^{*} p^{*}}{\left(h^{*}+k_{1}\right)^{2}}-i \omega_{0}^{n}\right) \frac{c_{2}}{a_{2}^{2}} .
$$

We also assume that $\left(q^{*}(s), q(\theta)\right)=1$. To obtain the value of $M$, from (A.9) we have

$$
\begin{aligned}
& \left(q^{*}(s), q(\theta)\right) \\
& =\bar{M}\left\{(1, \bar{\gamma})(1, \rho)^{T}\right. \\
& \left.\quad \quad-\int_{-1}^{0} \int_{\xi=0}^{\theta}(1, \bar{\gamma}) e^{-i(\xi-\theta) \omega_{0}^{n}} d \eta(\theta)(1, \rho)^{T} e^{i \xi \omega_{0}^{n}} d \xi\right\},
\end{aligned}
$$

then we choose

$$
\bar{M}=\left(1+\rho \bar{\gamma}+\frac{\rho c_{1} h^{*}}{h^{*}+k_{1}}\right)^{-1} e^{i \omega_{0}^{n}}
$$

such that $\left(q^{*}(s), q(\theta)\right)=0$ and $\left(q^{*}(s), q(\theta)\right)=1$. In other words, let $\Phi=(q(\theta), \bar{q}(\theta)), \Psi=\left(q^{*}(s), \bar{q}^{*}(s)\right)^{T}$, then $(\Psi, \Phi)=$ $I$, and $I$ is unit matrix.
Then the center subspace of model (A.4) is $P=\operatorname{span}\{q(\theta)$, $\bar{q}(\theta)\}$, and the adjoint subspace is $P^{*}=\operatorname{span}\left\{q^{*}(s), \bar{q}^{*}(s)\right\}$. Let $v=\left(v^{1}, v^{2}\right)^{T}$, where

$$
v^{1}=(1,0)^{T}, \quad v^{2}=(0,1)^{T} .
$$

Let $m \cdot v$ be defined by

$$
m \cdot v=m_{1} v^{1}+m_{2} v^{2}
$$

for $m=\left(m_{1}, m_{2}\right)^{T} \in([-1,0], X)$. Hence the center subspace of linear system (A.4) is given by $P_{C N} \mathscr{C}$, where

$$
\begin{gathered}
P_{C N} \phi=\Phi(\Psi,\langle\phi, v\rangle) \cdot v, \quad \phi \in \mathscr{C}, \\
P_{C N} \mathscr{C}=\{(q(\theta) z+\bar{q}(\theta) \bar{z}) \cdot v, z \in \mathbb{C}\},
\end{gathered}
$$

and $\mathscr{C}=P_{\mathrm{CN}} \mathscr{C} \oplus P_{S} \mathscr{C}$, where $P_{S} \mathscr{C}$ is the stable subspace.

From [17], we know that the infinitesimal generator $A_{U}$ of linear model (A.4) satisfies $A_{U} \psi=\dot{\psi}(\theta)$; moreover $\psi \in$ $\operatorname{dom}\left(A_{U}\right)$ if and only if

$$
\begin{gathered}
\dot{\psi}(\theta) \in \mathscr{C}, \quad \psi(0) \in \operatorname{dom}(\Delta), \\
\dot{\psi}(\theta)(0)=\tau_{0} \Delta \psi(0)+\tau_{0} L_{0}(\psi) .
\end{gathered}
$$

First we define the coordinate to describe the center manifold at $\alpha=0$; from center manifold we have

$$
\begin{aligned}
w(t, \theta) & =w(z(t), \bar{z}(t), \theta) \\
& =w_{20}(\theta) \frac{z^{2}}{2}+w_{11}(\theta) z \bar{z}+w_{02}(\theta) \frac{z^{2}}{2}+\cdots .
\end{aligned}
$$

The flow of model (A.2) in the center manifold can be written as follows:

$$
U_{t}=\Phi(z, \bar{z})^{T} \cdot v+w(z, \bar{z})
$$

where

$$
\begin{aligned}
\dot{z} & =i \omega_{0}^{n} z+q^{*}(0)\left\langle F\left(\Phi(z, \bar{z})^{T} \cdot v+w(z, \bar{z}), 0\right), v\right\rangle \\
& =i \omega_{0}^{n} z+g(z, \bar{z})
\end{aligned}
$$

with

$$
\begin{aligned}
g(z, \bar{z}) & =q^{*}(0)\left\langle F\left(\Phi(z, \bar{z})^{T} \cdot v+w(z, \bar{z}), 0\right), v\right\rangle \\
& =g_{20} \frac{z^{2}}{2}+g_{11} z \bar{z}+g_{02} \frac{\bar{z}^{2}}{2}+g_{21} \frac{z^{2} \bar{z}}{2}+\cdots .
\end{aligned}
$$

Let

$$
\begin{array}{r}
f^{1}=\left(H+h^{*}\right)\left(a_{1}-b\left(H+h^{*}\right)\right) \\
-\frac{c_{1}\left(H+h^{*}\right)\left(P(t-1)+p^{*}\right)}{k_{1}+H+h^{*}}, \\
f^{2}=\left(P+p^{*}\right)\left(a_{2}-\frac{c_{2}\left(P+p^{*}\right)}{k_{2}+H+h^{*}}\right) .
\end{array}
$$


From above equations, we have

$$
=\bar{M}\left(\begin{array}{c}
\rho\left(f_{110}^{1}+\bar{\gamma} f_{110}^{2}\right)+\rho e^{-i \omega_{0}^{n}}\left(f_{101}^{1}+\bar{\gamma} f_{101}^{2}\right) \\
+\rho^{2} e^{-i \omega_{0}^{n}}\left(f_{011}^{1}+\bar{\gamma} f_{011}^{2}\right) \\
+\frac{f_{200}^{1}+\bar{\gamma} f_{200}^{2}}{2}+\frac{\rho^{2}\left(f_{020}^{1}+\bar{\gamma} f_{020}^{2}\right)}{2} \\
+\frac{\rho^{2} e^{-2 i \omega_{0}^{n}}\left(f_{002}^{1}+\bar{\gamma} f_{002}^{2}\right)}{2}
\end{array}\right),
$$

$g_{11}(\theta)$

$$
=\bar{M}\left(\begin{array}{c}
\rho\left(f_{110}^{1}+\bar{\gamma} f_{110}^{2}\right)+\bar{\rho}\left(f_{110}^{1}+\bar{\gamma} f_{110}^{2}\right) \\
+\bar{\rho} e^{i \omega_{0}^{n}}\left(f_{101}^{1}+\bar{\gamma} f_{101}^{2}\right)+\rho e^{-i \omega_{0}^{n}}\left(f_{101}^{1}+\bar{\gamma} f_{101}^{2}\right) \\
+\bar{\rho} \rho e^{-i \omega_{0}^{n}}\left(f_{011}^{1}+\bar{\gamma} f_{011}^{2}\right)+\bar{\rho} \rho e^{i \omega_{0}^{n}}\left(f_{011}^{1}+\bar{\gamma} f_{011}^{2}\right) \\
+\left(f_{200}^{1}+\bar{\gamma} f_{200}^{2}\right)+\rho \bar{\rho}\left(f_{020}^{1}+\bar{\gamma} f_{020}^{2}\right)+\rho \bar{\rho}\left(f_{011}^{1}+f_{011}^{2}\right)
\end{array}\right),
$$

$\frac{g_{02}}{2}$

$$
=\bar{M}\left(\begin{array}{c}
\bar{\rho}\left(f_{110}^{1}+\bar{\gamma} f_{110}^{2}\right)+\bar{\rho} e^{-i \omega_{0}^{n}}\left(f_{101}^{1}+\bar{\gamma} f_{101}^{2}\right) \\
+\bar{\rho}^{2} e^{-i \omega_{0}^{n}}\left(f_{011}^{1}+\bar{\gamma} f_{011}^{2}\right) \\
+\frac{f_{200}^{1}+\bar{\gamma} f_{200}^{2}}{2}+\frac{\bar{\rho}^{2}\left(f_{020}^{1}+\bar{\gamma} f_{020}^{2}\right)}{2} \\
+\frac{\bar{\rho}^{2} e^{2 i \omega_{0}^{n}}\left(f_{002}^{1}+\bar{\gamma} f_{002}^{2}\right)}{2}
\end{array}\right),
$$

$\frac{g_{21}}{2}$

$$
=\bar{M}\left(\begin{array}{c}
\left(f_{110}^{1}+\bar{\gamma} f_{110}^{2}\right)\left(\rho w_{11}^{1}(0)+\frac{\bar{\rho} w_{20}^{1}(0)}{2}\right. \\
\left.+\frac{w_{20}^{2}(0)}{2}+w_{11}^{2}(0)\right) \\
+\left(f_{101}^{1}+\bar{\gamma} f_{101}^{2}\right)\left(\rho e^{-i \omega_{0}^{n}} w_{11}^{1}(0)+\frac{\bar{\rho} w_{20}^{1}(0) e^{i \omega_{0}^{n}}}{2}\right. \\
\left.+\frac{w_{20}^{2}(-1)}{2}+w_{11}^{2}(-1)\right) \\
+\left(f_{011}^{1}+\bar{\gamma} f_{011}^{2}\right)\left(\rho e^{-i \omega_{0}^{n}} w_{11}^{2}(0)+\frac{\bar{\rho} w_{20}^{2}(0) e^{i \omega_{0}^{n}}}{2}\right. \\
\left.+\bar{\rho} \frac{w_{20}^{2}(-1)}{2}+w_{11}^{2}(-1) \rho\right) \\
+\frac{1}{2}\left(f_{200}^{1}+\bar{\gamma} f_{200}^{2}\right)\left(2 w_{11}^{1}(0)+w_{20}^{1}(0)\right) \\
+\frac{1}{2}\left(f_{020}^{1}+\bar{\gamma} f_{020}^{2}\right)\left(2 w_{11}^{2}(0) \rho+w_{20}^{2}(0) \bar{\rho}\right) \\
+\frac{1}{2}\left(f_{002}^{1}+\bar{\gamma} f_{002}^{2}\right)\left(2 w_{11}^{2}(-1) e^{-i \omega_{0}^{n}} \rho\right. \\
\left.+w_{20}^{2}(-1) \bar{\rho} e^{-i \omega_{0}^{n}}\right)
\end{array}\right) .
$$

From [17], we can know that $w(z, \bar{z})$ satisfies

$$
\dot{w}=A_{U} w+H(z, \bar{z}),
$$

where

$$
\begin{aligned}
H(z, \bar{z}) & =H_{20}(\theta) \frac{z^{2}}{2}+H_{11}(\theta) z \bar{z}+H_{02}(\theta) \frac{\bar{z}^{2}}{2}+\cdots \\
& =X_{0} F\left(U_{t}, 0\right)-\Phi\left(\Psi,\left\langle X_{0} F\left(U_{t}, 0\right), v\right\rangle\right) \cdot v .
\end{aligned}
$$

Again we write near the origin on $C_{0}$

$$
\dot{w}=\dot{z} w_{z}+\dot{\bar{z}} w_{\bar{z}}
$$

By comparing (A.21) and (A.25) we get

$$
\begin{gathered}
\left(A_{U}-2 i \omega_{0}^{n}\right) w_{20}(\theta)=-H_{20}(\theta), \\
A_{U} w_{11}(\theta)=-H_{11}(\theta) .
\end{gathered}
$$

When $-1 \leq \theta<0, H(z, \bar{z})=-\Phi(\theta) \Psi(0)\left\langle F\left(U_{t}, 0\right), v\right\rangle \cdot v$. Therefore, for $-1 \leq \theta<0$,

$$
\begin{aligned}
& H_{20}(\theta)=-\left[g_{20} q(\theta)+\bar{g}_{02} \bar{q}(\theta)\right] \cdot v, \\
& H_{21}(\theta)=-\left[g_{21} q(\theta)+\bar{g}_{12} \bar{q}(\theta)\right] \cdot v,
\end{aligned}
$$

and for $\theta=0, H(z, \bar{z})(0)=F\left(U_{t}, 0\right)-\Phi\left(\Psi,\left\langle F\left(U_{t}, 0\right), v\right\rangle\right) \cdot v$, then we obtain

$$
\begin{aligned}
& H_{20}(0) \\
& =-\left[g_{20} q(0)+\bar{g}_{02} \bar{q}(0)\right] \cdot v \\
& +\tilde{\tau}\left(\begin{array}{c}
\left(\rho\left(f_{110}^{1}\right)+\rho e^{-i \omega_{0}^{n}}\left(f_{101}^{1}\right)+\rho^{2} e^{-i \omega_{0}^{n}}\left(f_{011}^{1}\right)\right. \\
\left.+\frac{f_{200}^{1}}{2}+\frac{\rho^{2} f_{020}^{1}}{2}+\frac{1}{2} \rho^{2} e^{-2 i \omega_{0}^{n}} f_{002}^{1}\right) \\
\left(\begin{array}{c}
\rho \bar{\gamma} f_{110}^{2}+\rho e^{-i \omega_{0}^{n}}\left(\bar{\gamma} f_{101}^{2}\right)+\rho^{2} e^{-i \omega_{0}^{n}}\left(\bar{\gamma} f_{011}^{2}\right) \\
\left.+\frac{\bar{\gamma} f_{200}^{2}}{2}+\frac{\bar{\gamma} \rho^{2} f_{020}^{2}}{2}+\frac{1}{2} \rho^{2} e^{-2 i \omega_{0}^{n}} \bar{\gamma} f_{002}^{2}\right)
\end{array}\right),
\end{array}\right.
\end{aligned}
$$

$H_{11}(0)$

$$
\begin{aligned}
& =-\left[g_{11} q(0)+\bar{g}_{11} \bar{q}(0)\right] \cdot v \\
& +\widetilde{\tau}\left(\begin{array}{l}
\left(f_{110}^{1}(\bar{\rho}+\rho)+f_{101}^{1}\left(\bar{\rho} e^{i \omega_{0}^{n}}+\rho e^{-i \omega_{0}^{n}}\right)\right. \\
+f_{011}^{1}\left(\rho \bar{\rho} e^{i \omega_{0}^{n}}+\rho \bar{\rho} e^{-i \omega_{0}^{n}}\right)+f_{200}^{1} \\
\left.+f_{020}^{1} \rho \bar{\rho}+f_{002}^{1} \rho \bar{\rho}\right)
\end{array}\right. \\
& \left(f_{110}^{2}(\bar{\rho}+\rho)+f_{101}^{2}\left(\bar{\rho} e^{i \omega_{0}^{n}}+\rho e^{-i \omega_{0}^{n}}\right)\right. \\
& +f_{011}^{2}\left(\rho \bar{\rho} e^{i \omega_{0}^{n}}+\rho \bar{\rho} e^{-i \omega_{0}^{n}}\right)+f_{200}^{2} \\
& \left.+f_{020}^{2} \rho \bar{\rho}+f_{002}^{2} \rho \bar{\rho}\right)
\end{aligned}
$$


By the definition of $A_{U}$, we have from (A.28)

$w_{20}(\theta)=\frac{i g_{20}}{\omega_{0}^{n}} q-(0) e^{i \theta \omega_{0}^{n}} \cdot v-\frac{i g_{02}}{3 \omega_{0}^{n}} \bar{q}(0) e^{-i \theta \omega_{0}^{n}} \cdot v+E e^{2 i \theta \omega_{0}^{n}}$,

where $E=\left(E^{1}, E^{2}\right) \in R^{2}$ is a constant vector.

Similarly, we get

$$
w_{11}(\theta)=-\frac{i g_{11}}{\omega_{0}^{n}} q(0) e^{i \theta \omega_{0}^{n}} \cdot v+\frac{i \bar{g}_{11}}{\omega_{0}^{n}} \bar{q}(0) e^{-i \theta \omega_{0}^{n}} \cdot v+\widetilde{E},
$$

where $\widetilde{E}=\left(\widetilde{E}^{1}, \widetilde{E}^{2}\right) \in R^{2}$ is a constant vector.

Combining (A.5) and (A.28), we obtain

$$
H_{20}(0)=2 i \omega_{0}^{n} \tilde{\tau} w_{20}(0)-\tilde{\tau} D \Delta w_{20}(0)-\tilde{\tau} L\left(w_{20}(\theta)\right),
$$

therefore

$$
\begin{aligned}
& \left(\begin{array}{c}
\left(\rho\left(f_{110}^{1}\right)+\rho e^{-i \omega_{0}^{n}}\left(f_{101}^{1}\right)+\rho^{2} e^{-i \omega_{0}^{n}}\left(f_{011}^{1}\right)\right. \\
+\frac{f_{200}^{1}}{2}+\frac{\rho^{2} f_{020}^{1}}{2}+\frac{1}{2} \rho^{2} e^{-2 i \omega_{0}^{n}} f_{002}^{1}
\end{array}\right) \\
& \left.\begin{array}{c}
\left(\rho \bar{\gamma} f_{110}^{2}+\rho e^{-i \omega_{0}^{n}}\left(\bar{\gamma} f_{101}^{2}\right)+\rho^{2} e^{-i \omega_{0}^{n}}\left(\bar{\gamma} f_{011}^{2}\right)\right. \\
\left.+\frac{\bar{\gamma} f_{200}^{2}}{2}+\frac{\bar{\gamma} \rho^{2} f_{020}^{2}}{2}+\frac{1}{2} \rho^{2} e^{-2 i \omega_{0}^{n}} \bar{\gamma} f_{002}^{2}\right)
\end{array}\right) \\
& =g_{20} q(\theta)+\bar{g}_{02} \bar{q}(\theta)+2 i \omega_{0}^{n} w_{20}(\theta) \\
& -\int_{-1}^{0} d \eta(\theta) w_{20}(\theta) \\
& =-g_{20} q(0)+\frac{\bar{g}_{02} \bar{q}(0)}{3}+E 2 i \omega_{0}^{n} \\
& -\int_{-1}^{0} d \eta(\theta)\left[\frac{i g_{20}}{\omega_{0}^{n}} q(\theta)-\frac{i g_{02}}{3 \omega_{0}^{n}} \bar{q}(\theta)-E e^{2 i \theta \omega_{0}^{n}}\right] \\
& =E\left(2 i \omega_{0}^{n} I-\int_{-1}^{0} d \eta(\theta) e^{2 i \theta \omega_{0}^{n}}\right) \\
& =2 i E \omega_{0}^{n}\left(\begin{array}{ll}
1 & 0 \\
0 & 1
\end{array}\right) \\
& -\left(\begin{array}{cc}
-b h_{+}+\frac{c_{1} h_{+} p_{+}}{\left(h_{+}+k_{1}\right)^{2}} & \frac{-c_{1} h_{+}}{h_{+}+k_{1}} e^{2 i \omega_{0}^{n}} \\
\frac{a_{2}^{2}}{c_{2}} & -a_{2}
\end{array}\right) E \\
& =\left(\begin{array}{cc}
2 i \omega_{0}^{n}+b h_{+}-\frac{c_{1} h_{+} p_{+}}{\left(h_{+}+k_{1}\right)^{2}} & \frac{c_{1} h_{+}}{h_{+}+k_{1}} e^{2 i \omega_{0}^{n}} \\
-\frac{a_{2}^{2}}{c_{2}} & 2 i \omega_{0}^{n}+a_{2}
\end{array}\right) E .
\end{aligned}
$$

From the above equation we can find the values of $E^{1}$ and $E^{2}$. From (A.28) we have $\int_{-1}^{0} d \eta(\theta) w_{11}(\theta)=-H_{11}(\theta)$. Therefore

$$
\begin{aligned}
& \left(\begin{array}{l}
\left(f_{110}^{1}(\bar{\rho}+\rho)+f_{101}^{1}\left(\bar{\rho} e^{i \omega_{0}^{n}}+\rho e^{-i \omega_{0}^{n}}\right)\right. \\
\left.+f_{011}^{1}\left(\rho \bar{\rho} e^{i \omega_{0}^{n}}+\rho \bar{\rho} e^{-i \omega_{0}^{n}}\right)+f_{200}^{1}+f_{020}^{1} \rho \bar{\rho}+f_{002}^{1} \rho \bar{\rho}\right)
\end{array}\right. \\
& \left(f_{110}^{2}(\bar{\rho}+\rho)+f_{101}^{2}\left(\bar{\rho} e^{i \omega_{0}^{n}}+\rho e^{-i \omega_{0}^{n}}\right)\right. \\
& \left.+f_{011}^{2}\left(\rho \bar{\rho} e^{i \omega_{0}^{n}}+\rho \bar{\rho} e^{-i \omega_{0}^{n}}\right)+f_{200}^{2}+f_{020}^{2} \rho \bar{\rho}+f_{002}^{2} \rho \bar{\rho}\right) \quad \\
& =g_{11} q(0)+\bar{g}_{11} \bar{q}(0)-\int_{-1}^{0} d \eta(\theta) w_{11}(\theta) \\
& =g_{11} q(0)+\bar{g}_{11} \bar{q}(0) \\
& -\int_{-1}^{0} d \eta(\theta)\left[-\frac{i g_{11}}{\omega_{0}^{n}} q(0) e^{i \omega_{0}^{n} \theta}+\frac{i \bar{g}_{11}}{\omega_{0}^{n}} e^{-i \omega_{0}^{n} \theta}+\widetilde{E}\right] \\
& =-\int_{-1}^{0} d \eta(\theta) \widetilde{E} \\
& =\left(\begin{array}{cc}
b h_{+}-\frac{c_{1} h_{+} p_{+}}{\left(h_{+}+k_{1}\right)^{2}} & \frac{c_{1} h_{+}}{h_{+}+k_{1}} \\
-\frac{a_{2}^{2}}{c_{2}} & a_{2}
\end{array}\right) \widetilde{E} .
\end{aligned}
$$

In a similar manner we can compute the corresponding results in $\widetilde{E}^{1}$ and $\widetilde{E}^{2}$. Then $g_{21}$ can be determined. Based on the above analysis, we can see that each $g_{i j}$ can be determined by the parameters. Thus we can computer the following values which determine the direction and stability of bifurcating periodic orbits:

$$
\begin{gathered}
c_{1}(0)=\frac{i}{2 \omega_{0}^{n}}\left(g_{11} g_{20}-2\left|g_{11}\right|^{2}-\frac{\left|g_{02}\right|^{2}}{3}\right)+\frac{g_{21}}{2}, \\
\mu=-\frac{\operatorname{Re}\left(c_{1}(0)\right)}{\operatorname{Re}\left(\lambda^{\prime}(\widetilde{\tau})\right)}, \quad \beta_{2}=2 \operatorname{Re}\left(c_{1}(0)\right), \\
T_{2}=-\frac{\operatorname{Im}\left(c_{1}(0)\right)+\mu_{2} \operatorname{Im}\left(\lambda^{\prime}(\tilde{\tau})\right)}{\omega_{0}^{n} \widetilde{\tau}} .
\end{gathered}
$$

\section{Acknowledgments}

The authors would like to thank the anonymous referee for very helpful suggestions and comments which led to improvements of their original paper. This research was supported by Natural Science Foundation of Zhejiang Province (LY12A01014 and LQ12A01009), the National Basic Research Program of China (2012CB426510), and the Fund Project of Zhejiang Provincial Education Department (Y201223449 and Y201120383). 


\section{References}

[1] A. F. Nindjin, M. A. Aziz-Alaoui, and M. Cadivel, "Analysis of a predator-prey model with modified Leslie-Gower and Hollingtype II schemes with time delay," Nonlinear Analysis: Real World Applications, vol. 7, no. 5, pp. 1104-1118, 2006.

[2] P. H. Leslie, "Some further notes on the use of matrices in population mathematics," Biometrika, vol. 35, pp. 213-245, 1948.

[3] P. H. Leslie, "A stochastic model for studying the properties of certain biological systems by numerical methods," Biometrika, vol. 45, pp. 16-31, 1958.

[4] P. Aguirre, E. González-Olivares, and E. Sáez, “Two limit cycles in a Leslie-Gower predator-prey model with additive Allee effect," Nonlinear Analysis: Real World Applications, vol. 10, no. 3, pp. 1401-1416, 2009.

[5] P. Aguirre, E. González-Olivares, and E. Sáez, "Three limit cycles in a Leslie-Gower predator-prey model with additive Allee effect," SIAM Journal on Applied Mathematics, vol. 69, no. 5, pp. 1244-1262, 2009.

[6] F. Chen, L. Chen, and X. Xie, "On a Leslie-Gower predator-prey model incorporating a prey refuge," Nonlinear Analysis: Real World Applications, vol. 10, no. 5, pp. 2905-2908, 2009.

[7] X. Guan, W. Wang, and Y. Cai, "Spatiotemporal dynamics of a Leslie-Gower predator-prey model incorporating a prey refuge," Nonlinear Analysis: Real World Applications, vol. 12, no. 4, pp. 2385-2395, 2011.

[8] T. Faria, "Stability and bifurcation for a delayed predator-prey model and the effect of diffusion," Journal of Mathematical Analysis and Applications, vol. 254, no. 2, pp. 433-463, 2001.

[9] R. Yafia, F. El Adnani, and H. T. Alaoui, "Limit cycle and numerical similations for small and large delays in a predatorprey model with modified Leslie-Gower and Holling-type II schemes," Nonlinear Analysis: Real World Applications, vol. 9, no. 5, pp. 2055-2067, 2008.

[10] B. I. Camara and M. A. Aziz-Alaoui, "Dynamics of a predatorprey model with diffusion," Dynamics of Continuous, Discrete \& Impulsive Systems, vol. 15, no. 6, pp. 897-906, 2008.

[11] S. Ruan, "On nonlinear dynamics of predator-prey models with discrete delay," Mathematical Modelling of Natural Phenomena, vol. 4, no. 2, pp. 140-188, 2009.

[12] Y. Song, S. Yuan, and J. Zhang, "Bifurcation analysis in the delayed Leslie-Gower predator-prey system," Applied Mathematical Modelling. Simulation and Computation for Engineering and Environmental Systems, vol. 33, no. 11, pp. 4049-4061, 2009.

[13] G.-P. Hu and W.-T. Li, "Hopf bifurcation analysis for a delayed predator-prey system with diffusion effects," Nonlinear Analysis: Real World Applications, vol. 11, no. 2, pp. 819-826, 2010.

[14] W. Zuo and J. Wei, "Stability and Hopf bifurcation in a diffusive predatory-prey system with delay effect," Nonlinear Analysis: Real World Applications, vol. 12, no. 4, pp. 1998-2011, 2011.

[15] F. Dumortier, J. Llibre, and J. C. Artés, Qualitative Theory of Planar Differential Systems, Springer, Berlin, Germany, 2006.

[16] E. González-Olivares, J. Mena-Lorca, A. Rojas-Palma, and J. D. Flores, "Dynamical complexities in the Leslie-Gower predatorprey model as consequences of the Allee effect on prey," Applied Mathematical Modelling. Simulation and Computation for Engineering and Environmental Systems, vol. 35, no. 1, pp. 366-381, 2011.

[17] J. Wu, Theory and Applications of Partial Functional-Differential Equations, vol. 119, Springer, New York, NY, USA, 1996.
[18] Y. Kuang, Delay Differential Equations with Applications in Population Dynamics, vol. 191, Academic Press, New York, NY, USA, 1993.

[19] B. D. Hassard, N. D. Kazarinoff, and Y. H. Wan, Theory and Applications of Hopf Bifurcation, vol. 41, 1981.

[20] J. K. Hale, Functional Differential Equations, Springer, 1971. 


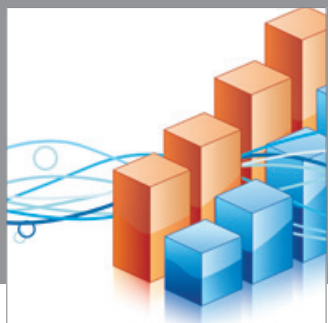

Advances in

Operations Research

mansans

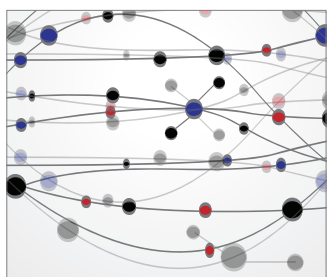

The Scientific World Journal
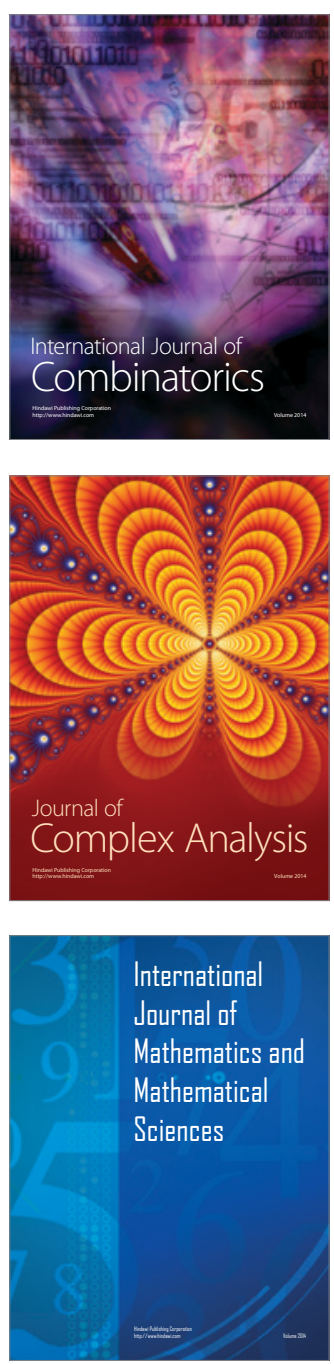
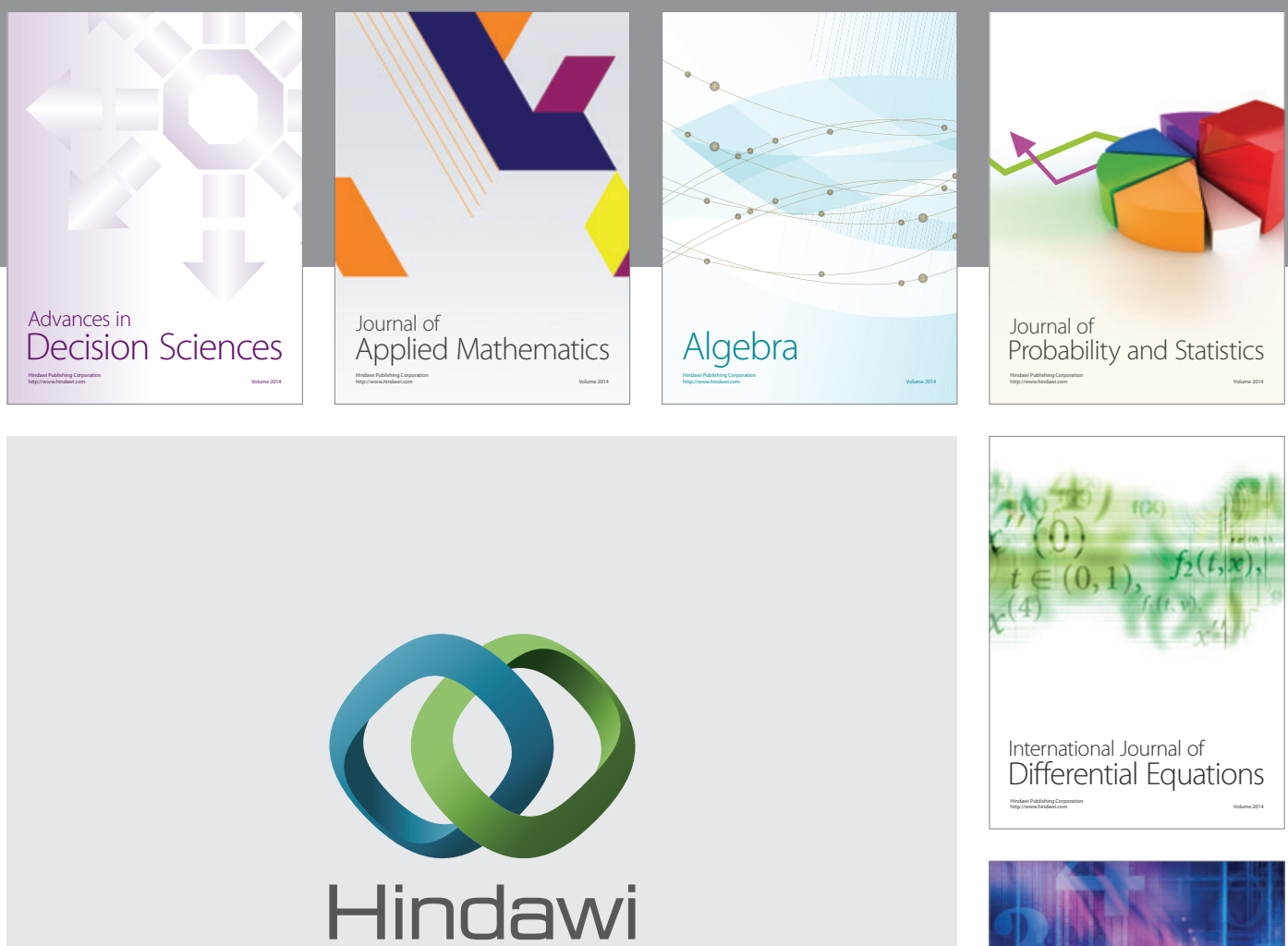

Submit your manuscripts at http://www.hindawi.com
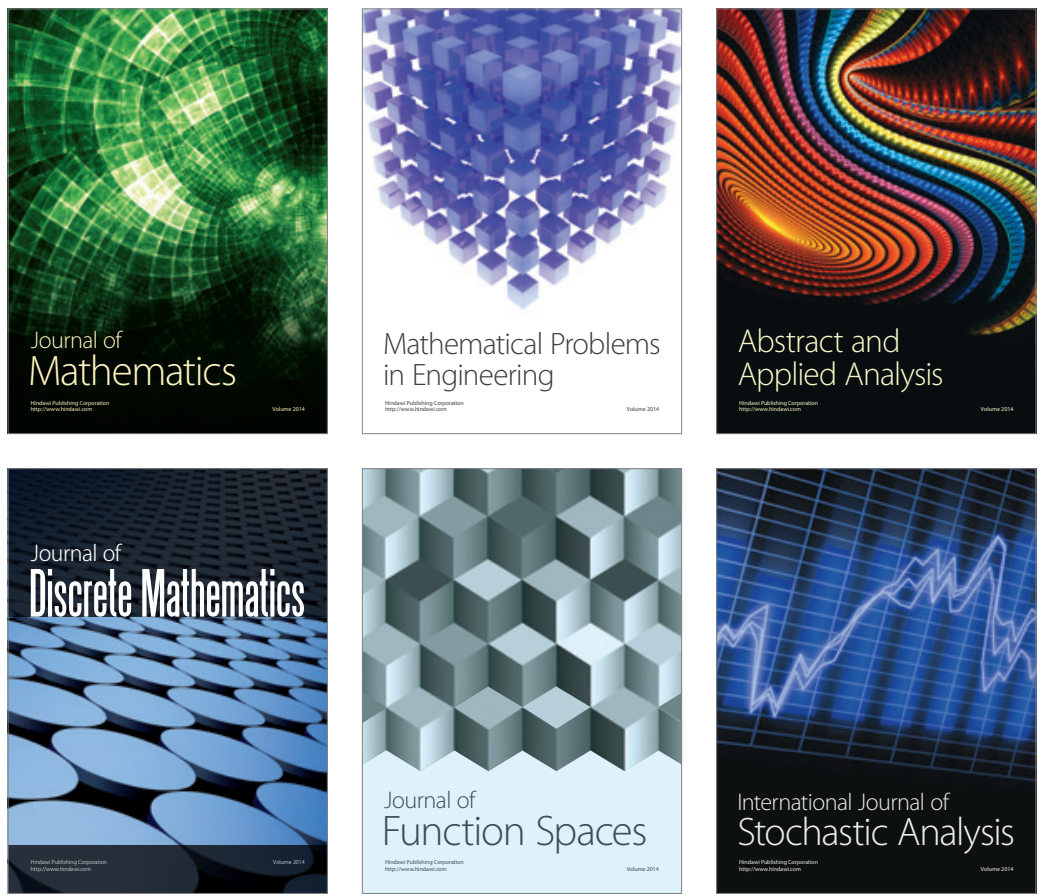

Journal of

Function Spaces

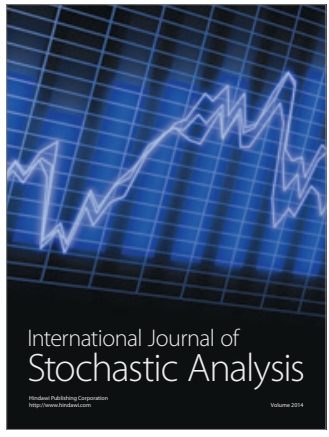

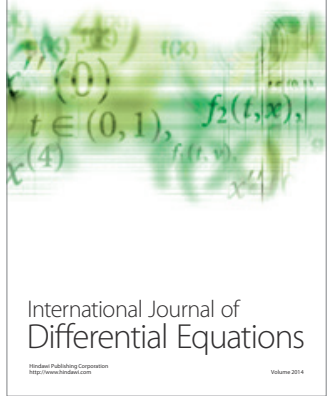
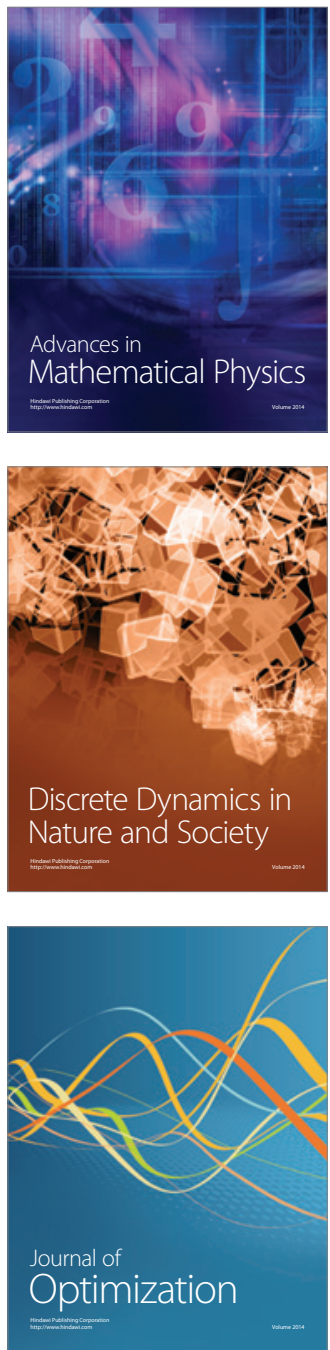